\title{
The Microbiology of Diabetic Foot Infections in Korea
}

\author{
Seung Tae Son, Seung-Kyu Han, Tae Yul Lee, Sik Namgoong, Eun-Sang Dhong \\ Department of Plastic Surgery, Korea University Guro Hospital, Seoul, Korea
}

\begin{abstract}
Background: In diabetic foot infection, causative pathogens differ in Western population as compared with Asian population. The aim of this study is to analyze microbiological data with respect to diabetic foot infection in Korea to help the choice adequate empirical antibiotics.

Methods: We enrolled 745 patients with diabetic foot infection, who were admitted to diabetic wound center of the Korea University Guro Hospital between January 2011 and December 2015. Deep tissue and/or bone biopsy cultures were taken during surgical debridement in the operating room and microbiological analysis was performed.

Results: Out of the 745 patients, 613 patients (82.2\%) had isolated causative organisms and 832 microbial isolates were identified. Gram-positive aerobic bacteria were isolated in 57.5\%, followed by Gram-negative aerobic bacteria (40.0\%), anaerobes (1.4\%), and fungus (1.1\%). MRSA found most frequently identified $(13.7 \%)$ in Gram-positive aerobes and Pseudomonas found most frequently identified (9.4\%) in Gram-negative aerobes.

Conclusion: Microbiologic analysis in Korea differs from the reports from Western population. We propose the need of our own guidelines based on microbiology of Korea for better treatment.
\end{abstract}

Keywords: Diabetic foot, Infection, Microbiology

\section{Introduction}

Diabetes mellitus is a serious health problem that is rapidly expanding worldwide. In 2000, according to the World Health Organization, at least 171 million people worldwide suffered from diabetes and due to increasing rates, this number is projected to double by 2030. ${ }^{1}$ Epidemiological studies suggest that $2.5 \%$ of diabetic patients develop diabetic foot ulcers each year and $15-25 \%$ during their lifetime. ${ }^{2}$ Diabetic foot ulcer is a result of various risk factors such as peripheral neuropathy, peripheral vascular disease, trauma, and impaired resistance to infection. ${ }^{3,4}$ Diabetic foot infection (DFI) is the most frequent and disastrous complication of the diabetic foot. ${ }^{5} \mathrm{DFI}$ is considered as a significant health and socioeconomic problem than any other diabetes-related complications and is the major cause of non-traumatic lower extremity amputations. ${ }^{6}$

When surgeons are confronted with diabetic foot infection in clinics, they choose the management strategy using antibiotics or surgical debridement. Current clinical guidelines for the management of DFI, according to Infectious Disease Society of America (IDSA), recommend empirical antimicrobial treatment until wound cultures ascertain the causative pathogens and their antibiotic susceptibility patterns, allowing targeted antibiologic treatment. ${ }^{7}$ The 3-5 days waiting period for results is the

\section{Original Article}

Received: May 9, 2017

Revised: May 16, 2017

Accepted: May 16, 2017

Corresponding author:

Seung-Kyu Han, M.D., Ph.D.

Department of Plastic Surgery, Korea

University Guro Hospital, 148 Gurodong-ro,

Guro-gu, Seoul 08308, Korea

Tel: +82-2-2626-3333

Fax +82-2-868-6698

E-mail: pshan@kumc.or.kr

No potential conflict of interest relevant to this article was reported.

This is an Open Access article distributed under the terms of the Creative Commons Attribution NonCommercial License (http://creativecommons.org/ licenses/by-nc/4.0/) which permits unrestricted noncommercial use, distribution, and reproduction in any medium, provided the original work is properly cited. (C) 2017 The Korean Wound Management Society 
critical period for initial infection management, during which most of the clinicians use relatively broad-spectrum empirical antimicrobial therapy, which covers most of the causative pathogens.

The previous studies derived from Western population provided a leading consensus that Gram-positive aerobes, particularly Staphylococcus aureus (S. aureus), are the predominant causative organisms in DFI.,9 Furthermore, the frequency of methicillin-resistant $S$. aureus (MRSA) has increased dramatically over the past 15 years. ${ }^{5,10-12}$ However, recent studies have demonstrated substantial variation in the etiology of DFI in different regions of the world. In contrast to Western population, investigations in Middle East, Asia, and Africa have found the most common isolates to be aerobic Gram-negative rods, particularly Pseudomonas aeruginosa. ${ }^{8,13-16}$ The prevalence of causative organisms in DFI may vary from region to region, and empirical antibiotic therapy should be optimized for local clinical microbiology. Therefore, the objective of this study was to analyze the microbiologic profile of DFI in Korea.

\section{Materials and Methods}

\section{Materials}

Medical records of 745 patients with diabetic foot ulcers, who were admitted and treated at the Diabetic Wound Center of Korea University Guro Hospital between January 2011 and December 2015, were reviewed. A complete medical history was obtained at the first visit. For analysis of the demographic and clinical characteristics, 8 variables such as age, gender, smoking history, dialysis, $\mathrm{HbA1C}$, wound duration, transcutaneous partial oxygen tension $\left(\mathrm{TcpO}_{2}\right)$ and previous use of antibiotics were investigated (Table 1). For patients who were admitted several times for different episodes, only the first admission period was included in this study. This study protocol was approved by the Institutional Review Board of Korea University Guro Hospital.

\section{Microbiological methods}

Only intraoperative samples collected from surgical procedures were included in the study. Surgical debridement was performed to remove all non-viable tissue from the wounds. Deep tissue and/or bone specimens were obtained and immediately stored in aseptic tube for culture. The specimens were sent to microbiologic laboratory in our hospital and incubated at $35^{\circ} \mathrm{C}$ for $24-48$ hours in Sheep blood agar plate.
Table 1. Patient demographics

\begin{tabular}{lc}
\hline Variable & Total $(\mathrm{n}=745)$ \\
\hline Age (year) & $62 \pm 12.1$ \\
Gender & \\
Male & $540(72.5 \%)$ \\
Female & $205(27.5 \%)$ \\
Smoking history & \\
Yes & $245(32.9 \%)$ \\
No & $500(67.1 \%)$ \\
Dialysis & \\
Yes & $176(23.6 \%)$ \\
No & $569(76.4 \%)$ \\
HbA1C (\%) & $8.0 \pm 1.8$ \\
Wound duration (week) & $19.1 \pm 8.3$ \\
Tcp0 (mmHg) & $34.6 \pm 20.7$ \\
Previous use of antibiotics & \\
Yes & $534(71.7 \%)$ \\
No & $211(28.3 \%)$ \\
\hline
\end{tabular}

$\mathrm{TcpO}_{2}$, Transcutaneous partial oxygen tension.

Furthermore, MacConkey II agar plate was used for aerobes and Chocolate agar plate for anaerobes.

\section{Results}

Out of the 745 patients enrolled in this study, 613 patients $(82.2 \%)$ had isolated causative organisms and 832 microbial isolates were identified which are presented in Table 2. Overall, 478 organisms (57.5\%) were Gram-positive and 333 organisms (40.0\%) were Gram-negative. In Gram-positive aerobes, MRSA was identified as the most commonly isolated organisms (13.7\%) followed by Enterococcus faecalis (12.6\%), Methicillin-sensitive S. aureus (MSSA) (12.5\%), Streptococcus agalactiae (6.5\%) and Methicillin-resistant Staphylococcus epidermidis (MRSE) (3.5\%). With reference to the Gram-negative organisms, Pseudomonas aeruginosa was identified as the most commonly isolated organisms (9.4\%) followed by Escherichia coli (7.2\%), Klebsiella pneumonia (3.2\%), Enterobacter cloacae (3.0\%), and Serratia marcescens (2.4\%).

\section{Discussion}

Diabetic foot infection is common and complex public health problem, particularly of importance in diabetes mellitus pa- 
Table 2. Microorganisms isolated from wound

\begin{tabular}{lc}
\hline Name of isolates & Number (\%) \\
\hline Gram positive aerobes & $478(57.5)$ \\
Staphylococcus aureus (MRSA) & $114(13.7)$ \\
Enterococcus faecalis & $105(12.6)$ \\
Staphylococcus aureus (MSSA) & $104(12.5)$ \\
Streptococcus agalactiae & $54(6.5)$ \\
Staphylococcus epidermidis (MRSE) & $29(3.5)$ \\
Corynebacterium striatum & $14(1.7)$ \\
Enterococcus faecium & $8(1.0)$ \\
Enterococcus faecium (VRE) & $5(0.6)$ \\
Others & $45(5.4)$ \\
Gram negative aerobes & $333(40.0)$ \\
Pseudomonas aeruginosa & $78(9.4)$ \\
Escherichia coli & $60(7.2)$ \\
Klebsiella pneumonia & $27(3.2)$ \\
Enterobacter cloacae & $25(3.0)$ \\
Serratia marcescens & $20(2.4)$ \\
Proteus mirabilis & $17(2.0)$ \\
Acinetobacter baumannii & $13(1.6)$ \\
Citrobacter freundii & $13(1.6)$ \\
Morganella morganii ssp. morganii & $12(1.4)$ \\
Stenotrophomonas maltophilia & $10(1.2)$ \\
Others & $58(7.0)$ \\
Oungaerobes & $9(1.1)$ \\
\hline
\end{tabular}

tients. ${ }^{17}$ To the best of our knowledge, this is the first singlecenter based microbiological study of DFI comprising of a large population in Korea (745 patients). Only deep tissue or bone culture obtained in operating room during surgical debridement were employed for this study, thus ensuring presence of reliable results on true pathogen of DFI.

The predominant causative microbiological organisms of DFI in Western population are Gram-positive aerobes, especially $S$. aureus, and the infection rate of MRSA has increased dramatically over the past 15 years. ., $, 10,12-14,18 \mathrm{~A}$ large multicenter study from the United States in 2008 discovered that $77 \%$ of DFIs were caused by Gram-positive aerobes, while $21.2 \%$ were caused by Gram-negative aerobes. ${ }^{9}$ Analogously, a more recent multi-center study involving microbiological
Table 3. Comparative analysis with a previous study

\begin{tabular}{lrr}
\hline Microorganism & 2007 & 2016 \\
\hline Gram positive aerobes & $76.4 \%$ & $57.5 \%$ \\
Staphylococcus aureus (MRSA) & $29.4 \%$ & $13.7 \%$ \\
Staphylococcus aureus (MSSA) & $15.6 \%$ & $12.5 \%$ \\
Enterococcus faecalis & $13.5 \%$ & $12.6 \%$ \\
Streptococcus agalactiae & $15.9 \%$ & $6.5 \%$ \\
Corynebacterium striatum & $2.0 \%$ & $17.0 \%$ \\
Gram negative aerobes & $33.3 \%$ & $40.0 \%$ \\
Pseudomonas aeruginosa & $7.8 \%$ & $9.4 \%$ \\
Escherichia coli & $7.3 \%$ & $7.2 \%$ \\
Klebsiella pneumonia & $6.9 \%$ & $3.2 \%$ \\
Enterobacter cloacae & $4.5 \%$ & $3.0 \%$ \\
Serratia marcescens & $4.3 \%$ & $2.4 \%$ \\
Acinetobacter baumannii & $2.3 \%$ & $1.6 \%$ \\
Fungal & $2.0 \%$ & $1.1 \%$ \\
Anaerobes & $0.0 \%$ & $1.4 \%$ \\
\hline
\end{tabular}

survey of DFI from Portugal revealed that $66 \%$ of DFIs were caused by aerobic Gram-positives, $19 \%$ by aerobic Gramnegatives, and $13.6 \%$ by obligate anaerobes. ${ }^{8}$

On the other hand, recent studies have demonstrated different etiological distribution of causative organisms of DFI in different regions of the world. ${ }^{13-15,18,19}$ Investigations from Middle East, Asia, and Africa have found the most common isolates to be aerobic Gram-negative rods, particularly Pseudomonas aeruginosa. ${ }^{8,13-16} \mathrm{~A}$ large study from Kuwait on DFI found that the major proportion of DFIs (51.2\%) were caused by aerobic Gram-negatives, while aerobic Gram-positives caused only $32.3 \%$ and obligate anaerobes only $15.3 \% .{ }^{18}$ Similarly, a recent study from India revealed that $51.4 \%$ of DFIs were caused by aerobic Gram-negatives, 33.3\% were caused by aerobic Gram-positives and 15.3\% were caused by anaerobic bacteria. ${ }^{20}$

Our study found that Gram-positive aerobes (57.5\%) were isolated more frequently than Gram-negative aerobes (40.0\%) and MRSA (13.7\%) was a predominant pathogen, but the proportion of MRSA was relatively small when compared to Western population (30.1-48.8\%)., ${ }^{9,21,22}$

This microbiological data was analyzed for comparing to our previous data obtained from same center in 2007, which estimated microbiological transition trend of diabetic foot infection. The distribution of microbial isolates between 2007 and 2016 is reported in Table 3. It can be seen from the data that the majority of identified organisms were Gram-positive 
(76.4\% in 2007, 57.9\% in 2016), and MRSA was most commonly isolated organisms (29.4\% in 2007, $13.7 \%$ in 2016) which exhibited a decreasing trend in contrary to the data reported in most of the Western population. The rate of MRSA as a cause of DFI has increased in Western population in recent years. ${ }^{12}$ In a study conducted in Manchester, UK in 2003 , the rate of MRSA isolates was $30.2 \%$, which was almost double the proportion of MRSA infected patients at their center 3 years back. ${ }^{10,11,20}$

On the other hand, Gram-negative aerobes $33.3 \%$ in 2007, 40.0\% in 2016) and Pseudomonas aeruginosa (7.8\% in $2007,9.4 \%$ in 2016) exhibited a similar trend of occurrence like other Asian population. Recent prospective study conducted in India showed the isolation from DFI as 62\% for all aerobic Gram-negatives, and 13\% specifically for Pseudomonas aeruginosa. ${ }^{23}$ This ratio is relatively higher than Western population. Published data in North America have reported the rate of Pseudomonas aeruginosa isolated from DFI as $4.5-6.5 \%{ }^{9,24,25}$ Some studies suggested the reasons for the high prevalence of DFI in Asian population as compared to Western population, which include hot climatic conditions leading to foot sweating, use of poor footwear, a high incidence of patient self-treatment with antimicrobials, frequent foot washing, and suboptimal perineal/hand hygiene. ${ }^{26}$

Current clinical guidelines, according to IDSA, for the management of DFI recommend empirical antibiotics classified by the fact whether Pseudomonas was isolated or not. The local rate of isolation of Pseudomonas is important for clinicians, because therapy directed for these species is usually different for other organisms

\section{Conclusion}

The results of microbiologic analysis in Korean DFI patients differ from the reports from Western population. Although MRSA was the most common causative organism, Pseudomonas had also been identified as a very important causative organism in Korea. These outcomes are expected to change empiric antibiotic therapy regimens for DFIs in Korea, as they need to be optimized for local clinical practice, and not only follow the guidelines of the Western population.

\section{References}

1. Wild S, Roglic G, Green A, et al. Global prevalence of diabetes: estimates for the year 2000 and projections for 2030. Di- abetes Care 2004;27:1047-53.

2. Reiber GE, Lipsky BA, Gibbons GW. The burden of diabetic foot ulcers. Am J Surg 1998;176:5s-10s.

3. Khanolkar MP, Bain SC, Stephens JW. The diabetic foot. Qjm 2008;101:685-95.

4. Noor S, Zubair M, Ahmad J. Diabetic foot ulcer--A review on pathophysiology, classification and microbial etiology. Diabetes Metab Syndr 2015;9:192-9.

5. Boulton AJ, Vileikyte L, Ragnarson-Tennvall G, et al. The global burden of diabetic foot disease. Lancet 2005;366: 1719-24.

6. van Battum P, Schaper N, Prompers L, et al. Differences in minor amputation rate in diabetic foot disease throughout Europe are in part explained by differences in disease severity at presentation. Diabet Med 2011;28:199-205.

7. Lipsky BA, Berendt AR, Cornia PB, et al. 2012 Infectious Diseases Society of America clinical practice guideline for the diagnosis and treatment of diabetic foot infections. Clin Infect Dis 2012;54:e132-73.

8. Mendes JJ, Marques-Costa A, Vilela C, et al. Clinical and bacteriological survey of diabetic foot infections in Lisbon. Diabetes Res Clin Pract 2012;95:153-61.

9. Lipsky BA, Holroyd KJ, Zasloff M. Topical versus systemic antimicrobial therapy for treating mildly infected diabetic foot ulcers: a randomized, controlled, double-blinded, multicenter trial of pexiganan cream. Clin Infect Dis 2008;47:1537-45.

10. Dang CN, Prasad YD, Boulton AJ, et al. Methicillin-resistant Staphylococcus aureus in the diabetic foot clinic: a worsening problem. Diabet Med 2003;20:159-61.

11. Eleftheriadou I, Tentolouris N, Argiana V, et al. Methicillin-resistant Staphylococcus aureus in diabetic foot infections. Drugs 2010;70:1785-97.

12. Tentolouris N, Jude EB, Smirnof I, et al. Methicillin-resistant Staphylococcus aureus: an increasing problem in a diabetic foot clinic. Diabet Med 1999;16:767-71.

13. Citron DM, Goldstein EJ, Merriam CV, et al. Bacteriology of moderate-to-severe diabetic foot infections and in vitro activity of antimicrobial agents. J Clin Microbiol 2007;45:2819-28.

14. Ramakant P, Verma AK, Misra R, et al. Changing microbiological profile of pathogenic bacteria in diabetic foot infections: time for a rethink on which empirical therapy to choose? Diabetologia 2011;54:58-64.

15. Shanmugam PMJ, Susan SL. The bacteriology of diabetic foot ulcers, with a special reference to multidrug resistant strains. J Clin Diagn Res 2013;7:441-5.

16. Uckay I, Aragon-Sanchez J, Lew D, et al. Diabetic foot infections: what have we learned in the last 30 years? Int J Infect Dis 2015;40:81-91.

17. Richard JL, Sotto A, Lavigne JP. New insights in diabetic foot infection. World J Diabetes 2011;2:24-32.

18. Al Benwan K, Al Mulla A, Rotimi VO. A study of the microbi- 
ology of diabetic foot infections in a teaching hospital in Kuwait. J Infect Public Health 2012;5:1-8.

19. Dezfulian A, Salehian MT, Amini V, et al. Bacteriological study of diabetic foot infections in an Iranian hospital. Iran Red Crescent Med J 2011;13:590-1.

20. Gadepalli R, Dhawan B, Sreenivas V, et al. A clinico-microbiological study of diabetic foot ulcers in an Indian tertiary care hospital. Diabetes Care 2006;29:1727-32.

21. Richard JL, Sotto A, Jourdan N, et al. Risk factors and healing impact of multidrug-resistant bacteria in diabetic foot ulcers. Diabetes Metab 2008;34:363-9.

22. Giordano P, Song J, Pertel P, et al. Sequential intravenous/ oral moxifloxacin versus intravenous piperacillin-tazobactam followed by oral amoxicillin-clavulanate for the treatment of complicated skin and skin structure infection. Int J Antimicrob Agents 2005;26:357-65.

23. KM M. The microbiological profile of diabetic foot infections. J Clin Diagn Res 2012;6:409-11.

24. Young $H$, Knepper B, Hernandez W, et al. Pseudomonas aeruginosa: an uncommon cause of diabetic foot infection. J Am Podiatr Med Assoc 2015;105:125-9.

25. Noel GJ, Bush K, Bagchi P, et al. A randomized, doubleblind trial comparing ceftobiprole medocaril with vancomycin plus ceftazidime for the treatment of patients with complicated skin and skin-structure infections. Clin Infect Dis 2008; 46:647-55.

26. Uckay I, Gariani K, Pataky Z, et al. Diabetic foot infections: state-of-the-art. Diabetes Obes Metab 2014;16:305-16. 